\title{
The Kinetics of Mycelial Growth
}

\author{
By A. L. KOCH \\ Department of Microbiology, Indiana University, Bloomington, Indiana 4740 , U.S.A.
}

(Received 6 August 1974; revised 20 December 1974)

\begin{abstract}
SUMMARY
Based on the assumption that mycelial growth follows the logistic growth law, formulae have been developed to express the growth of fungal colonies under a variety of geometric constraints. Analysis was done of Deppe's (1973) results on surface colony growth, where the mass of the colony grew exponentially during most colonial growth, and of Trinci's (1970) results on submerged 'pellet' growth, where the mass of the colony increased as the cube of time during most colony growth. In both cases the linear dimensions of the colony were increasing linearly while the mass was changing in these quantitatively different manners. It is concluded that these disparate growth behaviours result from different habits of growth; in two-dimensional colony growth a new region of space is invaded by an amount of mycelium small in proportion to the final 'carrying capacity' of the region, and in three-dimensional colony growth a region is invaded with an amount of mycelium almost equal to the region's final limiting mycelial mass. Thus, the types of growth law for colony mass which are applicable for a particular organism in a particular physical environment depend critically on the degree to which the invading hyphae initially occupy the space.
\end{abstract}

\section{INTRODUCTION}

Bacterial physiologists have focused attention on the state of balanced growth, in which every extensive property of the culture increases exponentially with the same growth-rate constant as every other. The growth of filamentous, branching organisms generally does not achieve this state fully or maintain it for very long. However, starting with any of a variety of propagules, after a lag phase or 'swelling-growth' phase, the total length of the hypha(e) in a colony increases geometrically for a short time, in the Malthusian sense. This has been observed for Chaetomium globosum (Plomley, 1959), Botrytis cinerea (Smith, 1924), and nine species of fungi studied by Trinci (1969, I97I $b$ ). It can be presumed that during much of this time other quantities, such as the dry weight, amount of DNA, number of nuclei, total content of a variety of enzymes, etc., should give the same exponential growth constant, corresponding to the same doubling time, as would total length.

One might also expect that after a fungus has grown and branched enough for a volume of space to be clearly delineated, the volume of the colony would increase exponentially with the same doubling time as any other extensive property. However, exponential increase in the physical size of the colony has only rarely been found; it has been observed for filamentous fungi growing in submerged shake-flask culture (Zalokar, 1959), and Trinci (1969) found an exponential increase in the radius of Aspergillus nidulans colonies which ended, however, when the radius reached $\mathrm{I} \mathrm{mm}$. Usually these dimensions do not increase exponentially, even at the earliest time in the development of the colony at which diameter, area or volume can be defined (see, for example, Trinci, 1970, 1971 $a$; Deppe, 1973).

Eventually, exponential growth (by any measure) must stop (Malthus, 1798), because 
of limitations in nutrient reserves, influx of nutrients, or available space. The purpose of this note is to point out that the Malthusian limit may be reached progressively for different parts of the colony in different ways, so that patterns of growth under different circumstances vary.

Under a variety of conditions and with a number of different organisms, mycelial and otherwise, the radius of the colony increases linearly with time during much of the total growth. This is because expansion of the colony is limited essentially by the rate of growth at the periphery. For mycelial growth this is limited by the rate of apical growth. It is those tips which are growing at right angles to the old surface, and therefore emigrating more rapidly into new territory, which establish the new frontier (Ryan, Beadle \& Tatum, 1943; Emerson, 1950). This rate of linear extension can be the same, irrespective of the age of the colony, if the conditions at the growing tip are time-invariant. This linear aspect of growth is also found in many other biological situations (see discussion in Koch, 1964). This reasoning about linear increase in linear dimension can be applied indefinitely to onedimensional growth in a growth tube, and for a limited time to two-dimensional surface colonies or three-dimensional submerged shake cultures. Obviously, the volume enclosed by the colony increases as the first, second or third power of time for these three configurations of growth, respectively, until growth is otherwise limited. The volume of the colony and its mass may break from exponential to power-law growth at different times in the development of the colony.

The observation that led to the present study was the finding of Deppe (1973) that for a number of fungi growing as surface colonies the net weight changed exponentially with time, and at the same time the radius changed linearly with time. She believes that the qualitative answer to these apparently contradictory modes of growth is that hyphal growth into uninhabited territory sparsely populates it, and only later fills it to full capacity. Under such conditions, extension of the colony in space is a measure of the maximum rate of exploration by the peripheral hyphae growing at right angles to the colony margin, while occupation of spaces within the limits of the mycelium simultaneously takes place by further branching. The latter may continue at a rate determined by the intrinsic rate of autocatalytic growth and local limitations until any particular volume of space carries its maximum capacity. The present contribution develops a quantitative theory to treat colonial-growth kinetics.

\section{MATHEMATICAL MODEL FOR COLONIAL GROWTH}

For the mathematical analysis, we start by assuming that after a neighbourhood has been inoculated by the penetration of a hyphal tip, further local growth in that region follows a logistic type of growth, as proposed for the numbers of individuals in populations by Verhulst (I838), Pearl (I927), Lotka (I925) and Volterra (I928). This law is the mainstay of today's theoretical ecology and should apply closely in cases where limitation of space is the most critical issue. It describes growth as being initially exponential, with a growth-rate constant which is characteristic of growth when the cellular elements are essentially independent of each other. Later, the specific growth rate decreases in proportion to the utilization of some factor in the environment, so that eventually the population is at its carrying capacity, $K$. If there is some upper asymptote for growth, then the differential equation for balanced growth is modified by the addition of a factor of a form that causes growth to become progressively slower. The new equation, with the factor shown in parentheses, is:

$$
\frac{\mathrm{d} N}{\mathrm{~d} t}=\lambda N\left(\frac{K-N}{N}\right)
$$


where $N$ is the number of organisms, $t$ is the time since there were $N_{0}$ organisms and $\lambda$ is the growth-rate constant for unrestricted growth. The solution is:

$$
N=N_{0} \mathrm{e}^{\lambda t}\left(\frac{K}{K-N_{0}+N_{0} \mathrm{e}^{\lambda t}}\right) .
$$

This derivation was conceived as applying to the number of individuals (see Odum, I97I), but if the same fundamental circumstance exists, it can apply equally to the number of cellular elements or to the amount of protoplasm in a mycelium.

In this familiar equation let us replace $N_{0}$ by $S$, which is then the initial mycelial density that inoculated a unit volume of space, $\mathrm{d} V$. Let us replace $N$, the number of individuals in the system under consideration, by $\mathrm{d} W / \mathrm{d} V$, where $\mathrm{d} W / \mathrm{d} V$ is the weight of protoplasm per unit volume element, and let us replace $t$ by $T-t$, where $T$ is the time elapsed since the growth of the colony was originally initiated, and now $t$ is the time at which the portion of the colony under consideration became infected. These replacements lead to:

$$
\mathrm{d} W=\frac{S K \mathrm{e}^{\lambda(T-t)}}{K-S+S \mathrm{e}^{\lambda(T-t)}} \mathrm{d} V ; T-t \geqslant 0 .
$$

Skellam (I95I) solved this problem by writing a differential equation which contained the differential formulation of the logistic growth law, instead of starting with its integrated form as done here. The solution he found was not simple, and recourse to tables of computed values was necessary. For this reason and because his solution only applies to a particular case, his type of solution has not been investigated further here.

Consider a colony growing with cylindrical symmetry on a surface; we can apply eqn (3) to various parts of the colony, and replace $\mathrm{d} V$ by $h \mathrm{~d} A$, where $\mathrm{d} A$ is an infinitesimal area and $h$ is a constant chosen so that $h K$ is the maximum carrying capacity per unit area; $h$ in certain cases is the maximum height that the colony either will attain before growth stops or can attain because of physical, spatial restrictions. For a cylindrical increment of growth, therefore:

$$
\mathrm{d} V=h \mathrm{~d} A=h \mathrm{~d}\left(\pi r^{2}\right)=2 \pi h r \mathrm{~d} r,
$$

where $r$ is the radius of the colony at the time that the volume element, $\mathrm{d} V$, was invaded.

We consider only the period when the colony is increasing at constant rate, $a$. It is assumed that the colony starts from a very small inoculum and that the lag phase of growth and the exponential phase of colony expansion can be neglected. Then $r=a t$ and $\mathrm{d} r=a \mathrm{~d} t$, and consequently eqn (4) can be re-expressed to eliminate the space variable, $r$, and replace it with a time variable, $t$, to become:

$$
\mathrm{d} V=2 \pi h a^{2} t \mathrm{~d} t \text { (for two dimensions). }
$$

Finally, on substituting this result into eqn (3) the weight of the colony at time $T$ is found to be given by the expression:

$$
W=\int_{0}^{T} \mathrm{~d} W=\int_{0}^{T} \frac{S K \mathrm{e}^{\lambda(T-t)}}{K-S+S \mathrm{e}^{\lambda(T-t)}} 2 \pi h a^{2} t \mathrm{~d} t,
$$

in which the only variable on the right-hand side within the integral is $t$.

In the derivation of this equation, it has been tacitly assumed that local growth in a neighbourhood is augmented by mycelial growth from other neighbourhoods, and lessened to the same degree by hyphal growth originating in the neighbourhood under consideration but projected into adjacent regions. As smaller and smaller volume elements are considered, these effects compensate more exactly. Obviously, in the very early phases of growth when the colony is small, emigration from a growing neighbourhood will exceed immigration, 
because there are more places to go to and fewer places from which to come. The increase in local biomass will then be slightly less than the predictions of eqns 3 and 6 .

Similar expressions may be written for growth in one or three dimensions by employing different expressions for $\mathrm{d} V$ :

$$
\mathrm{d} V=h b a \mathrm{~d} t \quad \text { (for one dimension), }
$$

where $b$ is the breadth of the growing column, and

$$
\mathrm{d} V=4 \pi a^{3} t^{2} \mathrm{~d} t \quad \text { (for three dimensions). }
$$

Except for the one-dimensional case, these expressions for $W$ cannot be analytically integrated without resorting to infinite series. We therefore set up computer programs to integrate them numerically. With the computer approach, the treatment can be extended to a variety of more realistic and special cases where $S, K, \lambda, a$, etc., vary as growth takes place or as the colony depletes local resources, or where the original inoculum is not infinitesimal. If these quantities are taken as constant, the shapes of the curves depend (apart from on the number of dimensions) on the chosen value of $K / S$. The choice of $\lambda$ merely defines a time scale, and the choice of $K, a$ and $h$ together with $\lambda$ merely determines a scale factor for the weight (in the two-dimensional case).

For illustrative purposes, Figs. I and 2 show the time course of growth of mycelial mass, calculated from eqn (6), for surface cylindrical colony growth in the idealized case where diameter expansion takes place at a uniform rate and the logistic law of growth applies in each locality. The ratios of $K / S$ were chosen to be 100 and ${ }^{1} 0^{4}$; with $K$ so much larger than $S$, a great deal of growth eventually takes place within a neighbourhood after it is initially inhabited. The $\log W$ versus $T$ curves (left-hand ordinate) are linear below the point where $K / S$ is approximately equal to $\mathrm{e}^{\lambda T}$, and curvilinear above it. Consequently, $K / S$ can be estimated roughly as $\mathrm{e}^{\lambda t}$ at the time when there is a bend in the logarithmic plot. However, because growth might slow for reasons other than the local achievement of the carrying capacity, this crude rule, or more careful curve fitting, will actually give too low a value for $K / S$; similarly, if the original inoculum is not infinitesimal compared with the final size, $K / S$ will again be underestimated.

Two other types of plots are shown in Figs. I and 2. The almost linear curves are for either the square root or cube root of weight scales shown on the right-hand ordinate. The examples were chosen so that both the phase in which $\log W$ increases linearly, and the later phase in which biomass growth is limited by the circular expansion of the surface colony so that the square root of the weight increases linearly, occur within the time range shown on the graph. It is perhaps surprising how extensive a region there is in which the cube root of weight increases linearly with time. There is no theory of which I am aware that predicts linearities of the cube root for the two-dimensional case, though there is for the three-dimensional case. It obviously arises in the two-dimensional case as a transition from the exponential to the square-root region.

The observations of Deppe (1973) that on membrane surfaces Schizophyllum commune, Morchella esculenta, Sordaria fimicola, Phycomyces blakesleeanus, and Agaricus bisporus grow exponentially in weight over a Io- to roo-fold range, yet grow linearly in diameter, implies that in all these cases $K / S$ is as large or larger than the observed factor of weight increase. For the case where $K / S$ is large, eqn (6) can be simplified and then analytically integrated, giving:

$$
W=\frac{2 \pi h a^{2} S K\left[\mathrm{e}^{\lambda T}-\lambda T+\mathrm{I}\right]}{(K-S) \lambda^{2}}
$$

assuming that we have started with an infinitesimal inoculum. The second and third terms 


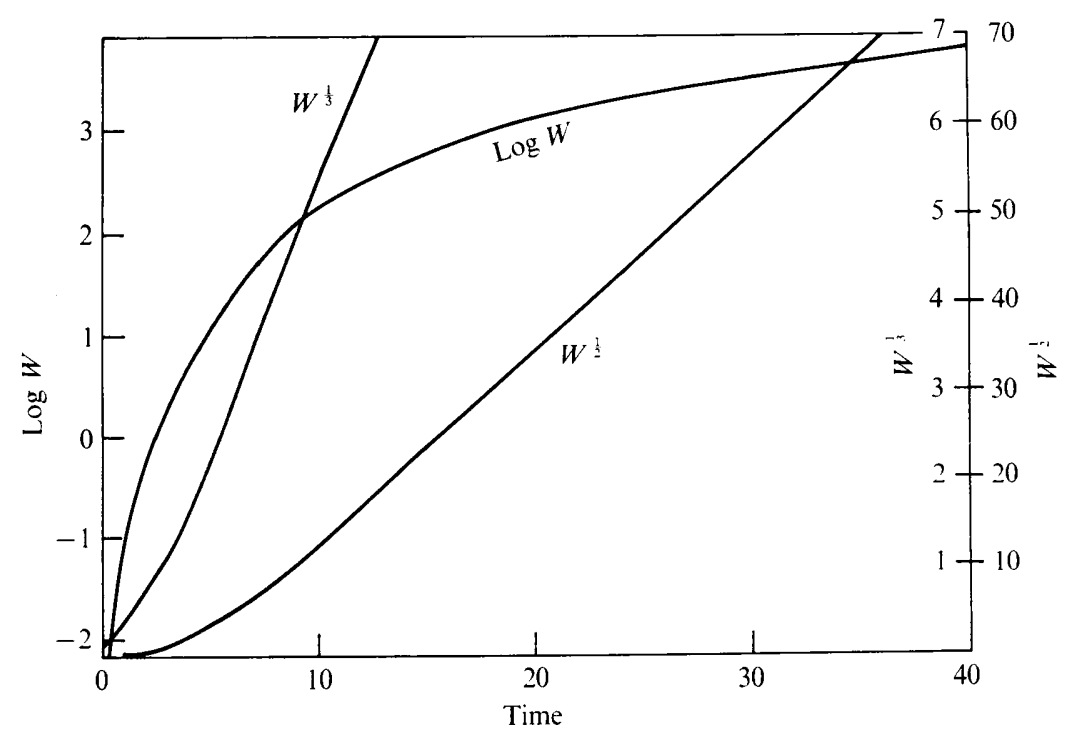

Fig. I. Kinetics of two-dimensional growth: $K / S=100$. The parameters $r, a$, and $2 \pi h a^{2}$ were arbitrarily chosen as I. $S$ was set at $0 \cdot I$ and $K$ at IO. Arbitrary units are used for time and weight.

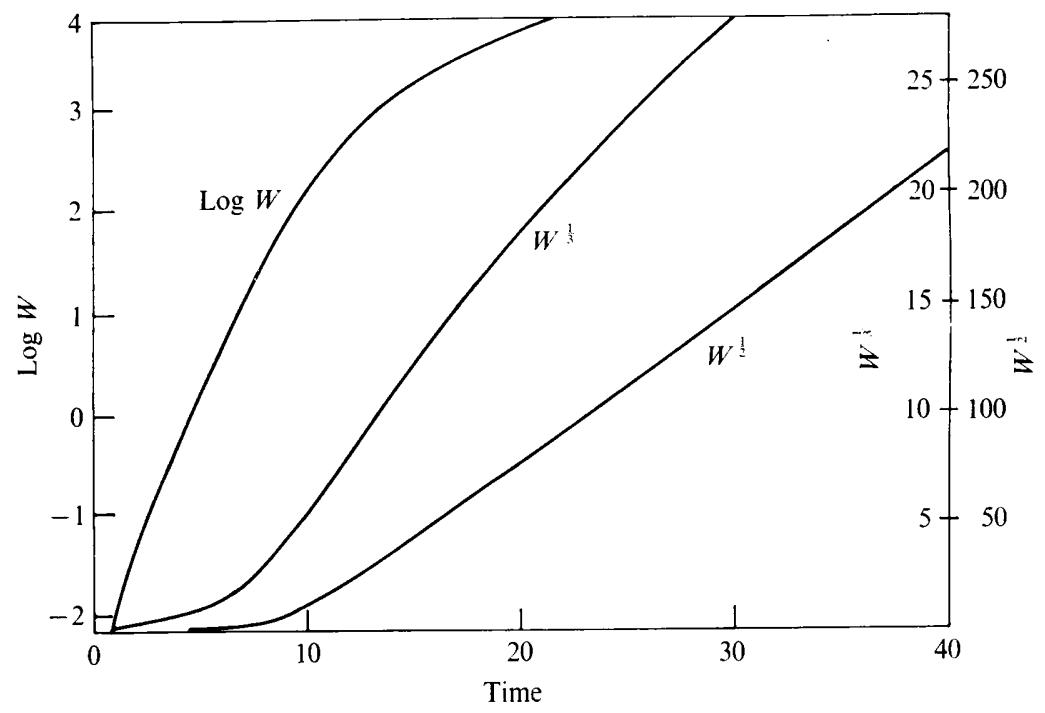

Fig. 2. Kinetics of two-dimensional growth: $K / S=10^{4}$. Parameters were chosen as in Fig. I except that $S$ was set at 0.01 and $K$ at 100. Arbitrary units are used for time and weight.

in the square brackets can be neglected as $\lambda T$ becomes larger than $\mathrm{I}$, and consequently the bracketed quantity quickly approaches $\mathrm{e}^{\lambda T}$. In fact, the term $(-\lambda T+\mathrm{I})$ would not be present in a more refined derivation.

As noted previously there is an approximation in the derivation which leads to an overestimate of biomass at early times. The same error is also present in eqn. (9). If we had corrected the local growth for immigration and emigration, the quantity in brackets in eqn (9) would have been $\mathrm{e}^{\lambda T}$ and the term $(-\lambda T+\mathrm{I})$ would not have been present at all. 


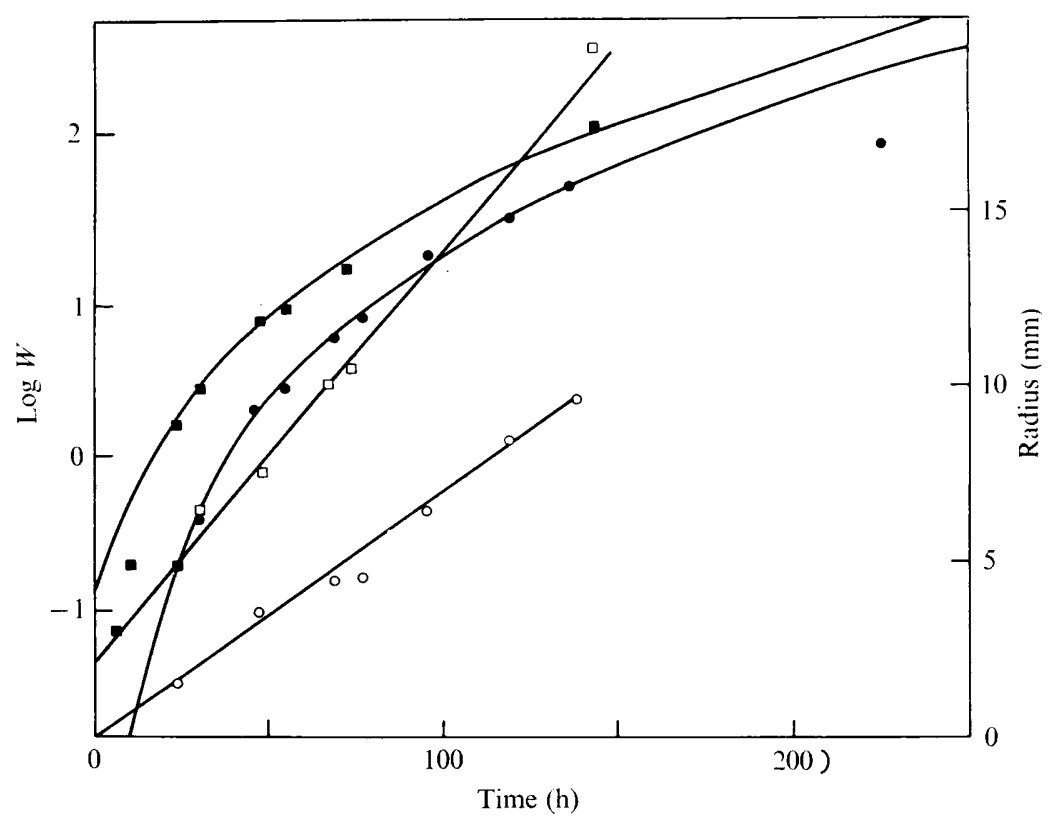

Fig. 3. The kinetics of three-dimensional growth. The points are taken from the work of Trinci (1970). The lines were fitted to these data and other auxiliary data from that paper by means of the computer program. The parameters $S$ and $K$ were fitted (see text). Solid symbols show the pellet dry weight, $W$, in $\mathrm{mg}$, at temperatures of $(\square) 37^{\circ} \mathrm{C}$ and $(\bullet) 25{ }^{\circ} \mathrm{C}$. Open symbols show the radius at (口) $37^{\circ} \mathrm{C}$ and (O) $25^{\circ} \mathrm{C}$.

If the full treatment were carried out the slope of the plot of the logarithm of biomass would be constant and equal to $2 \pi h a^{2} S K /(K-S) \lambda^{2}$ throughout.

This limiting form of the growth equation should be useful in that it allows an additional estimate of a quantity from actual experimental data which combines a number of the parameters of growth. This could only be done if a very small inoculum was used and if lag and pre-exponential phases of growth were brief. But, if these conditions were met, one could obtain $\lambda$ and $2 \pi h a^{2} S K /(K-S)^{2}$ from the slope and ordinal intercept values. If growth of the colony proceeds into a phase where the square root of mass changes linearly with time, the slope of such plots, $\left(\pi a^{2} h K\right)^{\frac{1}{2}}$, affords additional information. Then because one can measure $a$ and $h$ directly, it should be possible to estimate independently all the growth parameters of the theory: $S, K, h, \lambda$ and $a$. I have not yet seen any growth data in the literature where all the necessary measurements have been made.

For growth in three equivalent dimensions, the same computer programs with minor modification can be employed. In Fig. 3 some of the data obtained by Trinci (1970) are fitted. Trinci provides estimates of the exponential growth-rate constant, the linear weight of increase, and data from which the carrying capacity, $K$, can be approximated. By trial and error, the values of $S$ and $K$ were found that best fitted the data points at both the earliest and the latest time that the cultures grew according to the cube-root law. Data at both $37^{\circ} \mathrm{C}$ and at $25^{\circ} \mathrm{C}$ have been fitted. For the lower temperature, the radius of the colonies extrapolates to zero at zero time of incubation. For the upper temperature, sufficient growth had taken place in the inoculum culture to be equivalent to $20 \mathrm{~h}$ growth in the experimental period. This was taken into account in curve fitting. At $25{ }^{\circ} \mathrm{C}$, the best fit was obtained with $S=\mathrm{I} 2$ and $K=\mathrm{I} 8 \mathrm{pg} / \mu \mathrm{m}^{3}$; at $37{ }^{\circ} \mathrm{C}$, the best fit was obtained with $S=\mathrm{I} \cdot 5$ 
and $K=2.5 \mathrm{pg} / \mu \mathrm{m}^{3}$. The final pellet densities were 14.0 and $4.4 \mathrm{pg} / \mu \mathrm{m}^{3}$ at the two temperatures. The important result, needed for the main conclusion (below), is that no reasonable fit at all could be achieved if a value much greater than I was chosen for $K / S$.

\section{CONCLUSIONS}

Based on the assumption that a region has a maximum carrying capacity for fungal biomass, a flexible formulation has been developed here to describe the kinetics of growth of a large variety of mycelial organisms under various physical constraints.

So far this program has been applied to the observations of Deppe (1973), who found that a variety of fungal surface colonies simultaneously grew linearly in linear dimension and exponentially in weight. This is consistent with the assumption that the ratio of $K$ to $S$ is large $\left(\geqslant 10^{2}\right)$ in her example. On the other hand, Trinci's ( 1969 and $1971 a$ ) experiments show that the colonies of a variety of submerged shake cultures of fungi grow linearly in linear dimension and that the increase in weight is proportional to the cube of time for much of the growth phase. This implied that $K / S$ was only slightly greater than I, which was confirmed when his detailed published data for Aspergillus nidulans (Trinci, 1970) were fitted.

On a surface, growth can effectively proceed into a new area only while making contact with the nutritive surface. Only then can the space above the surface be occupied. Another aspect that also tends to make $K / S$ high is that the resources available below that surface are then only slowly mobilized.

For the pellet growing in shake culture a new domain is simultaneously invaded from the entire surface of pellet underlying it. Consequently, full occupancy is almost immediate. Moreover, because the volume is no longer adjacent to unoccupied territories, there is no source region that can supply nutrients as in the two-dimensional case, and $K / S$ approaches $\mathrm{I}$.

It is pertinent to consider the relationship between the present formulation and the growth zone proposed by Pirt (I966) in extension of the ideas of Emerson (1950) and Ryan et al. (1943), and supported by Trinci and many other workers. According to Pirt's concept, only the cortex, of fixed thickness $\Delta$, engages in growth in the pellet type of threedimensional growth. This accounts for the linear increase in radius and the linear increase in cube root of weight with time, because we can then write, if $l$ is the rate constant for linear growth of the cortex region:

$$
\frac{\mathrm{d} r}{\mathrm{~d} t}=l \Delta
$$

and because $W=4 \pi r^{3} \rho / 3$, where $\rho$ is the density, it follows that

Substituting for $r$ in eqn (I I) gives:

$$
\frac{\mathrm{d} W}{\mathrm{~d} t}=4 \pi r^{2} \Delta \rho l \text {. }
$$

$$
\frac{\mathrm{d} W}{\mathrm{~d} t}=4 \pi W^{\frac{2}{3}} \Delta \rho l\left(\frac{4}{3} \pi \rho\right)^{\frac{2}{3}}
$$

This can be integrated to yield:

$$
W^{\frac{1}{3}}=W_{0}^{\frac{1}{3}}+(4 \pi \rho / 3)^{\frac{1}{3}} \Delta l t .
$$

Thus this model, too, predicts a cube-root law for three-dimensional growth. In terms of the model proposed in this paper, the constant growth zone, $\Delta$, corresponds to that region behind the growing front that has not yet reached carrying capacity. However, in this model it is not proposed that growth is uniform within this zone as it is in Pirt's model. The present 
formulation should be more realistic because it allows for the growing area to shade into the non-growing area. The present model should be testable and predictive, if it is valid for colonial growth over ranges spanning more than one habit of growth.

The work has been supported by National Institutes of Health under grant AI 09337 and by the National Science Foundation under grant 32 I I 5 . I thank Carol Deppe, E. Janet Evans and A. P. J. Trinci for constructive comments.

\section{REFERENCES}

DEPPE, C. S. (1973). Protein degradation in Schizophyllum commune. Ph.D. thesis, University of Harvard, U.S.A.

Emerson, S. (1950). The growth phase in Neurospora corresponding to the logarithmic phase in unicellular organisms. Journal of Bacteriology 60, $22 \mathrm{I}-223$.

Косн, A. L. (1964). The growth of viral plaques during the enlargement phase. Journal of Theoretical Biology 6, 4I3-43I.

LotkA, A. V. (1925). Elements of Physical Biology. Baltimore: Williams and Wilkins. [Reprinted (I956) under title Elements of Mathematical Biology. New York: Dover.]

Malthus, T. R. (1798). An Essay on the Principle of Population. London: Johnson.

Odum, E. P. (197I). Fundamentals of Ecology, 3rd edn. Philadelphia: W. B. Saunders.

Pearl, R. S. (1927). The Biology of Population Growth. New York: A. E. Knopf.

PIRT, S. J. (I966). A theory of the mode of growth of fungi in the form of pellets in submerged culture. Proceedings of the Royal Society B r66, 369-373.

Plomley, N. J. B. (1959). Formation of the colony in the fungus Chaetomium. Australian Journal of Biological Science I2, 53-64.

Ryan, F. J., Beadle, G. W. \& Tatum, E. L. (I943). The tube method of measuring the growth rate of Neurospora. American Journal of Botany 30, 789-799.

SkellaM, J. G. (I95I). Random dispersal of theoretical populations. Biometrika 38, 189-2 I8.

Sмith, J. H. (1924). On the early growth rate of the individual fungus hypha. New Phytologist 23, 64-79.

TrincI, A. P. J. (I969). A kinetic study of the growth of Aspergillus nidulans and other fungi. Journal of General Microbiology 57, I I-24.

TrincI, A. P. J. (1970). Kinetics of the growth of mycelial pellets of Aspergillus nidulans. Archiv für Mikrobiologie 73, 353-367.

TrincI, A. P. J. (I97I $a$ ). Influences of the width of the peripheral growth zone on the radial growth rate of fungal colonies on solid media. Journal of General Microbiology 67, 325-344.

Trinci, A. P. J. (I97I $b$ ). Exponential growth of the germ tubes of fungal spores. Journal of General Microbiology 67, 345-348.

VERHUlST, P. F. (1838). Notice sur la loi que la population suit dans son accoissement. Correspondance Mathématique et Physique ro, I13-121. [Reprinted (1965), in translation, in Readings in Ecology, pp. 64-66, edited by E. J. Kormandy. Englewood Cliffs: Prentice-Hall.]

VOLTERRA, V. (1928). Variations and fluctuations of the number of individuals in animal species living together. As translated in Animal Ecology (1931), pp. 409-448, edited by R. N. Chapman. New York: McGrawHill.

Zalokar, M. (1959). Growth and differentiation of Neurospora hyphae. American Journal of Botany 46, $602-609$. 